physical activity through changes to the nursery environment. Feasibility and acceptability have been demonstrated through Randomised Controlled Trials (RCT) in the USA. This study examined the feasibility and acceptability of adapting the NAP SACC intervention for the UK.

Methods A feasibility cluster RCT in 12 nurseries with 24 year olds in the southwest region of England. Focus groups and interviews with Health Visitors (community children's nurses), nursery staff and parents informed adaptation of the intervention for the UK. The intervention comprised: two staff workshops on physical activity and nutrition; Health Visitor support to review nursery practices against 80 areas of best practice, set goals and make changes; a digital media-based home component. Measures were assessed at baseline and post-intervention: zBMI, accelerometer-measured physical activity and sedentary time, diet, child quality of life, health care usage, parental and nursery staff mediators and quality of nursery environment. Fidelity and acceptability were assessed through observation and interviews analysed via thematic analysis.

Results Formative work resulted in the following adaptations: inclusion of an oral health component; changes to confirm with UK guidance; specialist workshop facilitators; and development of the home component. 168 (37\%) eligible children were recruited from 12 nurseries. Interviews were completed with four Health Visitors, 17 nursery staff and 20 parents. The intervention was implemented with high fidelity, with two exceptions: one nursery did not implement the intervention due to staff workload; and the digital home component was used by just 12 (14\%) parents. Intervention acceptability was high. A mean of seven staff per nursery attended each workshop. The workshops and Health Visitor contact were highly valued. The mean number of goals set was eight. Nursery changes included: menu modifications, reducing portion sizes and sugary snacks, role modelling physical activity and eating, and active story telling. The trial design and methods were highly acceptable. Descriptive analysis of the outcomes will be available by September 2017.

Conclusion NAP SACC UK is feasible and acceptable with the exception of the home component; effectiveness should be tested through a full-scale RCT.

\section{P26 ASSOCIATIONS BETWEEN TYPOLOGIES OF NEIGHBOURHOOD ENVIRONMENTS AND ASSOCIATIONS WITH OBESITY: A CROSS-SECTIONAL STUDY}

${ }^{1,2} \mathrm{M}$ Hobbs $*,{ }^{2} \mathrm{C}$ Griffiths, ${ }^{3} \mathrm{M}$ Green, ${ }^{4} \mathrm{~J}$ Saunders, ${ }^{5} \mathrm{H} J$ Jordan, ${ }^{2} \mathrm{~J}$ McKenna. ${ }^{1}$ School of Social
and Health Sciences, Leeds Trinity University, Leeds, UK; ${ }^{2}$ Carnegie, Leeds Beckett
University, Leeds, UK; ${ }^{3}$ Geography and Planning, The University of Liverpool, Liverpool, UK;
${ }^{4}$ Carnegie, Leeds Beckett University; formerly, Rotherham Borough Council, Rotherham, UK;
${ }^{5}$ School of Health and Related Research (ScHARR), The University of Sheffield, Sheffield, UK

\subsection{6/jech-2017-SSMAbstracts. 128}

Background Recent research has demonstrated that neighbourhood features such as fast-food outlets and supermarkets may co-occur. However, little research has investigated the combined influences of both the built food and physical activity (PA) environments and associations with body mass index and obesity. This study aims to use latent class analysis within a large UK adult population to investigate associations between the combined environment and obesity.

Methods Cross-sectional, individual-level data $(n=22,889)$ from Wave 1 of The Yorkshire Health Study (2010-2012) were used. Body mass index (BMI) was calculated using selfreported height and weight; obesity $=\mathrm{BMI} \geq 30$. Neighbourhood was defined as a $2 \mathrm{~km}$ radial buffer; food outlets and physical activity facilities were sourced (2012) from Ordnance Survey Points of Interest (PoI) and categorised into 'fast-food', 'large supermarkets', 'convenience and other food retail outlets' and 'physical activity facilities'. Parks were sourced from Open Street Map. Latent class analysis (LCA) was conducted on these five environmental variables. Logistic regression was then conducted to predict obesity based on the five neighbourhood types identified within LCA. Models adjusted for age, gender, ethnicity, area-level deprivation and rural or urban classification of the neighbourhood.

Results A five-class solution fitted the dataset best and was interpretable. Neighbourhood typologies were labelled as; "low exposure" (19.0\% of study population); "moderate exposure" (33.3\%); "moderate PA, limited food" (12.2\%); "saturated" (13.6\%); "moderate PA, ample food" (21.2\%). For associations with obesity, those within the low exposure typology were chosen as the exposure because low exposure to physical activity environments have the potential to reduce physical activity behaviours and although more debatable poorer access to the food environment may result in poorer dietary intake. Compared to the low exposure, one typology showed lower odds of obesity ("saturated", $\mathrm{OR}=0.86[0.75,0.99]$ ) and one showed increased odds of obesity ("moderate exposure, $\mathrm{OR}=1.18$ [1.05,1.32].

Discussion Meaningful neighbourhood typologies were derived from a range of food and physical activity measures using latent class analysis which explained differences in obesity in large UK based sample of adults. This study suggests that neighbourhoods were not wholly unhealthy or healthy, they were characterised by neighbourhood features that are both health-promoting and health-constraining and this resulted in complex associations with obesity.

\section{P27 CAN WE BETTER CAPTURE LONGITUDINAL EXPOSURE TO THE NEIGHBOURHOOD ENVIRONMENT? A LATENT CLASS GROWTH ANALYSIS OF THE OBESOGENIC ENVIRONMENT IN NEW YORK CITY, 1990-2010}

${ }^{1} \mathrm{~N}$ Berger*, ${ }^{2}$ TK Kaufman, ${ }^{3} \mathrm{MDM}$ Bader, ${ }^{2} \mathrm{DM}$ Sheehan, ${ }^{4} \mathrm{~S} J$ Mooney, ${ }^{5} \mathrm{KM}$ Neckerman, ${ }^{2}$ AG Rundle, ${ }^{6} \mathrm{GS}$ Lovasi. ' ${ }^{1}$ Social and Environmental Health Research, London School of Hygiene and Tropical Medicine, London, UK; ${ }^{2}$ Department of Epidemiology, Columbia University Mailman School of Public Health, New York City, USA; ${ }^{3}$ Department of Sociology, Centre on Health, Risk and Society, American University, Washington, USA; ${ }^{4}$ Department of Epidemiology, School of Public Health, Harborview Injury Prevention and Research Centre, University of Washington, Seattle, USA; ${ }^{5}$ Columbia Population Research Centre, New York City, USA; ${ }^{6}$ Department of Epidemiology and Biostatistics, Dornsife School of Public Health, Urban Health Collaborative, Drexel University, Philadelphia, USA

\subsection{6/jech-2017-SSMAbstracts. 129}

Background The growing availability of (non-)commercial historical datasets opens a new avenue of research on how longterm exposure to the neighbourhood environment affects health. However, traditional tools for longitudinal analysis (e. g. mixed models) are limited in their ability to operationalise long-term exposure. This study aims to summarise longitudinal exposure to the neighbourhood using latent class growth analysis (LCGA). Using the National Establishment Time-Series (NETS) 1990-2010, we analysed the trajectory of change in New York City (NYC) in the number of unhealthy food businesses - a potential indicator of an obesogenic environment. 
Methods The NETS is a commercial dataset providing retail business information in the United States. NYC data were acquired for the period 1990-2010. Businesses were grouped into researcher-defined categories based on Standard Industrial Classification codes and other fields such as business name. All businesses were re-geocoded to ensure accurate localisation. We defined access to BMI-unhealthy businesses (characterised as selling calorie-dense foods such as pizza and pastries) as the total number of BMI-unhealthy businesses present in each NYC census tract $(n=2,167)$ in January of each year. We conducted LCGA in Mplus to identify census tracts with similar trajectories of BMI-unhealthy businesses. We used model fit statistics and interpretability to determine the number of classes. Using the final models, we assigned census tracts to latent classes. We predicted class membership with socio-demographic variables from the Census (population size, income, and ethnic composition) using multinomial logistic regressions and reported predicted probabilities with 95\% CI. Sensitivity analyses were undertaken.

Results The final models include 5 and 10 latent classes, respectively. The 5 -class solution indicates an overall increase in the number of BMI-unhealthy businesses over time and shows a pattern of fanning out: the higher the value in 1990, the greater the increase over time. Classes are associated with 1990 population size, income, proportion of Black residents (all $\mathrm{p}<0.001)$, proportion of Hispanic residents $(\mathrm{p}=0.033)$, and 1990-2010 change in population size and income $(\mathrm{p}<0.001)$. The 10-class solution identifies two pairs of classes with similar 1990 values, but different trajectories. Differences in those trajectories are associated with population size and ethnic composition $(\mathrm{p}<0.001)$.

Conclusion This study illustrates how LCGA contributes to the understanding of long-term exposure to the obesogenic environment. The technique can easily be applied to other aspects of the neighbourhood and to other geographies. When linked with health data, identified latent classes can be used to assess how longitudinal exposure to changing neighbourhoods affects health.

\section{P28 ASSOCIATION BETWEEN INDOOR TEMPERATURE AND GENERAL HEALTH IN ENGLISH ADULTS: A CROSS- SECTIONAL STUDY}

1,2 J Sutton-Klein*, 'A Moody, J Mindell. 'Institute of Epidemiology and Health, University
College London, London, UK; ${ }^{2}$ The Medical School, University of Sheffield, Sheffield, UK

10.1136/jech-2017-SSMAbstracts. 130

Background While the association between indoor temperature and health has been indirectly investigated using variables including self-reported cold homes and the presence of insulation as proxies for temperature, research using indoor temperature directly has been lacking. We hypothesised that lower indoor temperatures would be associated with increased odds of poor general health, which would be partially explained by socioeconomic and housing variables.

Methods We used data from the Health Survey for England collected between 2003 and 2014. The analytical sample consisted of 74735 participants aged over 16. Indoor temperature was approximated using a one-off reading of indoor air temperature in participants' homes. Self-reported general health was used for the main outcome, dichotomised into very good/good/fair vs. bad/very bad.
Using Stata, logistic regression models were built for the data, using maximum likelihood estimation. The complex survey design and weights were taken into account in the analysis. The influence of socioeconomic and housing factors was assessed by adjusting for these variables in the models.

Results The mean indoor temperature was $20.6^{\circ} \mathrm{C}$ (SE 0.09), which varied seasonally and regionally. Warmer indoor temperatures were associated with greater odds of poor general health. The age and sex-adjusted odds ratio (OR) of poor health for each degree increase in indoor temperature was 1.01 (95\% CI 1.01-1.02 P<0.01). Lower social class and lower education level were associated with warmer temperatures and poorer health. The OR decreased after adjusting for these confounding variables. Higher income was associated with warmer temperatures and fairer health. After adjusting for both the suppressor and confounding variables, the OR for poor health for each degree increase in temperature has a statistically significant increase by $19 \%$ to 1.02 (95\% CI $1.01-$ $1.03 \mathrm{P}<0.01)$.

Conclusion Our analysis unexpectedly showed an association between high indoor temperature and poor health. The relationship between socioeconomic factors and indoor temperature was complicated, with different socioeconomic variables having different directions of association with indoor temperature. Further research is needed to understand the temporality and direction of the association. The research is timely given the potential for data from increasingly prevalent smart thermostats.

\section{P29 RACIAL INEQUALITIES IN DENTAL SERVICE UTILISATION AMONGST MIDDLE-AGED BRAZILIAN ADULTS}

HM Constante*, G Tsakos, RG Watt. Department of Epidemiology and Public Health, University College London, London, UK

\subsection{6/jech-2017-SSMAbstracts. 131}

Background Dental service utilisation is important as it helps to alleviate pain and suffering, and access to dental treatment. International evidence has shown that racial/ethnic minority groups are less likely to use dental services compared to the White majority population. In Brazil, where the concept of colour/race is used to classify the population, recent research has assessed colour/race inequalities in dental service use amongst the elderly population, but evidence is scarce for other age groups. Therefore, the overall aim of this research is to investigate the differences between colour/race groups (White versus Pardo and Black) in the use of dental service in a national sample of 35 to 44 year-old adults in Brazil taking into consideration the role of individual-level characteristics. Methods Data from 7902 adults aged 35 to 44 years from the Brazilian National Oral Health Survey (SB Brazil 2010) was used in this study. The survey collected data using an interviewer-administrated questionnaire and clinical examinations. Dependent variables were the time since last dental visit (less than a year, or a year or more), reason for the last dental visit (prevention/check-up, pain or extraction, or treatment), and type of service used (public, or private). The main exploratory variable was self-reported colour/race (White, Pardo, or Black) measured according to the Brazilian Institute of Geography and Statistics (IBGE). Covariates were sex, level of education, family income, satisfaction with teeth/mouth, self-reported need for treatment, self-reported dental pain in 